\title{
Notre expérience en coenseignement
}

Auteures

Nadia Gray, Commission scolaire des grandes seigneuries, Canada, nadia.gray@csdgs.net

Julie Jeannotte, Commission scolaire des grandes seigneuries, Canada, julie.jeanotte@csdgs.net 


\section{REVUE HYBRIDE DE L'ÉDUCATION}

\section{Résumé}

Nous présentons, dans cet article notre expérience de coenseignement du français à des groupes d'élèves à risque de l'école Louis-Philippe-Paré (Châteauguay). Nous décrivons les outils, les mesures et les méthodes de travail que nous avons jugées efficaces pour soutenir les élèves. Nous évoquons également les conditions à réunir pour une saine collaboration entre coenseignants, et formulons quelques souhaits et recommandations afin d'optimiser cette méthode d'enseignement.

Mots-clés : coenseignement, soutien, partenariat, enseignant-ressource, volontariat, formation continue 


\section{$\&$}

\section{REVUE HYBRIDE DE L'ÉDUCATION}

\section{Mise en contexte}

Nous sommes enseignantes de français en troisième secondaire à l'école Louis-Philippe-Paré à Châteauguay. Cet établissement fait partie de la commission scolaire des Grandes-Seigneuries en Montérégie. II s'agit d'une école publique d'environ 1800 élèves. Depuis trois ans, nous enseignons dans les classes de mesures d'aide où les élèves du « régulier » bénéficient du coenseignement. Ces classes ont vu le jour pour optimiser la mesure enseignant-ressource octroyée par le MELS depuis 2006.

Au départ, la fonction d'enseignant-ressource consistait surtout à offrir un support supplémentaire aux élèves des classes ordinaires à risque ou présentant différents troubles liés à l'apprentissage. Cette mesure d'aide supplémentaire prenait principalement deux formes : le soutien aux élèves en classe ou l'intervention hors classe, en sous-groupe de besoins.

Au fil du temps, ce mode de fonctionnement a démontré certaines limites. La première relève du caractère ponctuel de la présence de l'enseignant-ressource en classe. II ne connaissait pas suffisamment les élèves, ce qui permettait difficilement d'établir une relation signifiante qui favorise l'apprentissage. Le peu de fréquence dans les interventions de la ressource a conduit à un manque de continuité dans le suivi des élèves, la capacité à soutenir l'apprentissage dans une matière donnée et l'impossibilité de percevoir si les élèves s'amélioraient ou non. De même, la faible connaissance des diagnostics et des besoins particuliers des élèves avait un impact sur la pertinence des interventions. Cela se soldait par le fait que les élèves préféraient poser leurs questions à leur enseignant habituel, l'enseignant-ressource n'ayant pas été présent au moment des explications données en classe. En termes de consignes ou de règles, des contradictions entre les exigences de l'enseignant et celle de l'enseignantressource étaient fréquentes. Enfin, comme les périodes d'enseignementressource étaient préétablies, il était souvent difficile de combiner la planification des cours avec le moment où la ressource se présentait en classe. Par exemple, lors d'une production écrite qui se déroulait sur trois ou quatre cours, la présence de la ressource était parfois inutile, car elle coïncidait avec la mise en texte individuelle plutôt qu'à des moments interactifs où les élèves auraient pu bénéficier de rétroactions. Insatisfaits de l'efficacité de cette mesure, certains enseignants ont cherché des solutions afin d'optimiser le service.

\section{Élèves ciblés}

Depuis quatre ans, en $3^{e}$ secondaire, nous privilégions le coenseignement pour les groupes d'élèves les plus à risque. Les élèves de ces classes sont ciblés pour leurs faibles résultats en français, c'est-à-dire qu'ils ont obtenu une moyenne entre $57 \%$ et $65 \%$ à la fin de leur deuxième secondaire. Certains sont médicamentés et ont différents diagnostics: 


\section{$\&$}

\section{REVUE HYBRIDE DE L'ÉDUCATION}

TDAH, dyslexie, dysorthographie, dyspraxie, TSA, etc. Beaucoup de ces élèves doivent utiliser des ordinateurs en classe. En collaboration avec la direction adjointe et la direction de l'école, nous avons fait un plan d'intervention pour notre classe de mesures d'aide en français. Nous avons pris des mesures concrètes pour offrir des conditions gagnantes à tous nos élèves. Les mesures mises en place permettent aux élèves :

- d'utiliser un Lexibook en période d'écriture en classe (32 Lexibook ont été achetés par la direction) ;

- de bénéficier d'une période de 75 minutes fixe dans l'horaire pour utiliser le laboratoire informatique ;

- de se voir accorder un tiers de temps supplémentaire pour faire les exercices et les évaluations, s'ils le souhaitent ;

- de recevoir un enseignement sur l'utilisation du logiciel Antidote qui est disponible sur tous les ordinateurs de l'école;

- d'accéder aux textes pour les examens de lecture une semaine à l'avance ;

- de rencontrer, pendant une période par cycle de neuf jours, une orthopédagogue qui vérifie que les élèves font une utilisation efficiente de leurs outils de correction électroniques ;

- une offre de récupération abondante (au moins cinq jours sur neuf) sur l'heure du dîner. Un micro-onde a été installé en classe pour permettre aux élèves de dîner avec nous et d'éviter la perte de temps à la cafétéria ;

- un suivi serré avec les parents lors de chaque début d'étape, pour leur donner toutes les dates d'évaluation et de remise de travaux.

Ces mesures permettent véritablement de soutenir les élèves dans l'apprentissage du français. D'ailleurs, nos élèves se disent satisfaits du service offert. En effet, plusieurs se disent chanceux d'avoir deux profs, deux visions, deux façons différentes de recevoir l'enseignement des notions vues en classe. Ils sentent qu'ils ont un soutien supplémentaire. Nous voyons parfois poindre un nouveau sentiment de compétence chez certains élèves qui comprennent davantage. Ils disent apprécier le dynamisme des échanges et le climat de classe convivial qui règne. Nous sommes convaincues que nos élèves terminent leur année scolaire avec plus de moyens pour optimiser leur potentiel en français.

Toutefois, une ombre demeure au tableau. Ces mesures d'aide causent beaucoup de controverses au sein du département de français de notre école. Certains collègues voient ces mesures comme étant trop facilitantes pour les élèves et pensent qu'elles ne les préparent pas à l'évaluation d'écriture de cinquième secondaire. Deux philosophies se confrontent, et sous le prétexte de l'autonomie professionnelle, toutes les mesures mises en place ne sont pas reconduites. Un bris s'effectue dans les pratiques et entrave la progression des apprentissages des élèves lorsqu'ils changent de niveau. Cela se perçoit par le désengagement et les résultats plus faibles qu'obtiennent une partie des élèves qui ont bénéficié 


\section{8}

\section{REVUE HYBRIDE DE L'ÉDUCATION}

du soutien offert en $3^{e}$ secondaire. Nous sommes d'avis que la continuité doit nécessairement être planifiée sans quoi les efforts déployés se trouvent invalidés.

\section{Fonctionnement de classe}

Lorsque nous coenseignons, nous privilégions le modèle où l'une des deux enseignantes donne son cours et l'autre agit en tant que soutien. L'enseignante-ressource répond aux questions des élèves et enrichit les explications en apportant un complément d'information. Elle agit également comme soutien à la gestion de tout ce qui est plus technique, soit remettre des documents à toute la classe, écrire des dates importantes au tableau, chercher des mots dans le dictionnaire, parler en privé à un élève, etc. II arrive aussi que nous fassions un enseignement partagé, particulièrement au moment de faire la phrase du jour (une approche pédagogique en ce qui a trait à l'enseignement de la grammaire, que nous préconisons). Enfin, lorsque nous le jugeons nécessaire, nous séparons le groupe en deux sous-groupes pour travailler différents contenus ou pour pouvoir nous attarder sur des problématiques différentes.

Lorsque nous planifions ces activités, nous nous assurons qu'il y a une constance dans notre approche. Les élèves se sont habitués et ne font plus la différence entre une enseignante ou l'autre. D'ailleurs, chaque année, l'une d'entre nous accueille des stagiaires et les élèves réagissent bien à cette troisième personne qui s'ajoute au groupe. Le coenseignement permet aussi à l'étudiant en formation de voir plusieurs modèles d'enseignement dans une même classe et de valider la pertinence de certaines pratiques.

\section{Conditions pour une saine collaboration}

Faire du coenseignement, c'est un peu comme être en couple. Pour que cela fonctionne, il doit y avoir une certaine harmonie entre les deux protagonistes. Il est vrai que chacun amène sa personnalité, sa façon d'enseigner et sa vision des choses. Par contre, il est essentiel de développer une certaine complémentarité. Les deux personnalités doivent pouvoir travailler de concert et développer un dynamisme qui leur est propre. Par exemple, un individu avec une personnalité plus rigide ou qui est plus exigeant, jumelé à quelqu'un de beaucoup plus souple, pourrait vivre un sentiment de frustration s'il adopte une attitude fermée. Dans cette perspective, pour être coenseignant, il faut pouvoir faire preuve de souplesse, être capable d'ouverture et de réciprocité, et savoir s'adapter à la façon de faire de son partenaire. II est important que les deux enseignants aient une vision pédagogique semblable. Ainsi, il faut aimer travailler avec des élèves en difficulté, être patient avec eux, savoir les apprécier, les encourager et avoir le désir de les amener plus loin. 


\section{$\&$}

\section{REVUE HYBRIDE DE L'ÉDUCATION}

L'humour et l'humilité sont également des qualités requises pour être un bon coenseignant. Ces qualités permettent de relativiser les situations vécues et de réfléchir sur sa pratique. Cela nécessite toutefois une bonne dose de confiance en soi pour analyser ses forces et ses défis, entre collègues.

Planifier à deux des séquences didactiques, c'est se donner le cadeau de se confronter aux nouvelles idées, de voir les choses d'un autre angle dans le but de répondre aux besoins des élèves et de leurs intérêts. Pour cela, il faut consacrer du temps à communiquer et à s'organiser. L'expérience vécue fait que nous nous connaissons mieux. Nous avons appris à nous compléter et nous croyons être devenues de meilleures enseignantes.

\section{Coenseignement : améliorations souhaitées dans les années à venir}

La direction d'établissement joue nécessairement un rôle important dans un tel modèle de service. Elle doit croire à la pertinence du coenseignement et favoriser son bon déroulement, notamment en consacrant une partie de son budget à la création de binômes dans les différentes disciplines au programme.

Durant les prochaines années, il serait primordial de maintenir le service du coenseignement pour les élèves en classe ordinaire ayant des difficultés ou des problématiques particulières. Ces élèves sont de plus en plus nombreux et représentent une partie importante des élèves qui fréquentent notre école. Parmi les souhaits formulés, le nombre d'élèves par groupe serait idéalement limité à 20 élèves. Afin d'assurer la continuité, les outils ainsi que les stratégies d'apprentissage utilisés en troisième secondaire devraient être les mêmes durant les deux années suivantes. Dans un esprit de collégialité, une collaboration régulière et un meilleur arrimage des services offerts par l'orthopédagogue, le psychologue et l'éducateur spécialisé, contribueraient à l'optimisation du service. De plus, des formations sur le coenseignement devraient être offertes pour les enseignants qui démontrent de l'intérêt.

En ce sens, favoriser la diffusion des expériences vécues entre collègues s'avère primordial pour stimuler d'autres enseignants à s'intéresser à ce modèle de service et en assurer la pérennité. En somme, il est impératif de planifier ce service en termes de continuité, si l'on souhaite contribuer à la réussite des élèves.

\section{En conclusion, quelques recommandations}

Le coenseignement est une belle expérience pour les enseignants et il est bénéfique aux élèves. Toutefois, il n'y a pas de miracle qui s'opère. II faut travailler beaucoup et différemment. Parmi les considérations, en voici quelques-unes qui devraient être prise en compte de façon prioritaire : 


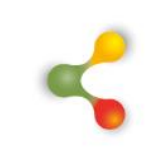

\section{REVUE HYBRIDE DE L’ÉDUCATION}

a) les enseignants en coenseignement doivent être volontaires; b) ils doivent aussi se concerter avant le choix de tâche et se choisir comme partenaires; c) il est bénéfique qu'ils s'inscrivent activement dans une démarche de formation continue; d) le rôle de chaque enseignant devrait être clarifié au départ et des réajustements faits tout au long de l'année scolaire; e) des rencontres fréquentes avec les autres membres de l'équipe-école devraient être organisées pour assurer une cohésion entre les actions des divers personnels impliqués. À cet égard, procéder à l'analyse des résultats obtenus par les élèves permettrait de cibler les stratégies pédagogiques à mettre en œuvre. Enfin, f) il est nécessaire que la direction exerce son leadership pédagogique afin d'assurer la qualité du service de coenseignement.

L'ajout d'une ressource supplémentaire en classe mérite d'être encadré et une concertation avec les intervenants du milieu améliorerait cette offre de service aux élèves. 